\title{
Hipercalciuria idiopática. Diagnóstico diferencial
}

\author{
M.C. Francisco Cano Sch. ' ; M.C. Eugenio Rodríguez S. ${ }^{1}$; M.C. María Angela Detucchi B. ${ }^{\prime}$; \\ M.C. Eduardo Wolff $P{ }^{1}$
}

\section{Idiopathic hypercalciuria. Urinary calcium/creatinine ratios}

In 50 children with hematuria or urolithiasis and idiopathic hypercalciuria, and in 15 control children, urinary calcium/creatinine concentration rates were measured after fasting and after calcium loading. Patients were classified into two groups depending on the results of an orally administered cakium loading test. Children wete considered to have absortive hypercalciuria $(42 \%)$ when they had low fasting urinary calcium/creatinine concentration ratio $(<0.21)$, and a large increase of this index after calcium administration $(>0.28)$. Patients were labeled as renal hypercalciuria $(32 \%)$ if they had high fasting urinary calcium/creatinine concentration ratio $(>0,21)$, and variable increases of it after calcium overload. A third group of children $(26 \%)$, were not classifiable by means of this test. Ouc data support the contention that this simple ambulatory test is very aseful in the diagnostic workup of idiopathic hypetcalciuria.

(Key words: hematuria, urinary calculi, hypercalciuria, idiopathic)

La hipercalciuria idiopática ha cobrado creciente interés en los últimos años al demostrarse su relación etiológica con la hematuria en porcentajes que oscilan entre 25 y $40 \%$ de los grupos estudiados ${ }^{1-4}$. Por otra parte, su importancia en el estudio de las litiasis urinarias es reconocida desde que se determinara que entre 40 y $75 \%$ de los cálculos compuestos por calcio son secundarios a este trastorno. ${ }^{4-6}$.

EJ estudio sistemático de esta enfermedad fue iniciado por Pak en 1975, con su clásica descripción de una prueba que permitio su clasificación en los subgrupos absortivo y renal? con elementos fisiopatológicos propios, de relevante importancia para el enfoque terapéutico ${ }^{7-11}$. Esta clasificación ha sido discutida por distintos autores que no han confirmado los hallazgos originales, postulándose formas intermedias a partir de una teoría unificadora de la hipercalciuria iđiopática, en que los fenómenos absortivos serían conse-

1. Unidad de Nefrología y Hemodiálisis, Hospital Luis Calvo Mackenna. cuencia de las alteraciones metabólicas de origen renal ${ }^{6,12-15}$.

Este estudio tuvo como propósito evaluar prospectivamente en un grupo de pacientes hipercalciúricos la posibilidad de subdividirlos en los tipos absortivo y renal, según su comportamiento en una prueba de restricción y sobrecarga de calcio, basada en modelos ya descri$\operatorname{tos}^{6,7,10,16,17}$, con el objeto de avanzar hacia un tratamiento racional de esta afección.

\section{MATERIAL Y METODOS}

Se evaluaron 50 pacientes hipercalciúricos detectados entre niños que consultaron por hematuria, litiasis o ambas, que en una muestra aistada de orina en ayunas tenían un índice calcio/creatinina mayor a 0,21 o calciuria en orina de $24 \mathrm{~h}$ superior a $4 \mathrm{mg} \cdot \mathrm{kg} \cdot \mathrm{dí}$ (valores considerados como límite máximo normal en niños). Se evaluaron como controles 15 pacientes sin hipercalciuria. Su distribución por sexo y promedio de edad es descrita en la tabla 1. Las formas secundarias de hipercalcjuria se excluyeron a través de anamnesis y exarnen físico detallados y el estudio sistemático de normalidad de los exámenes de orina completa, urocul- 
tivo, proteinuria, gases sanguíneos, electrolitos en plasma y orina, calcemia, fosfemia, fosfatasas alcalinas, tadiografía de abdomen simple, ecotomografía renal, pielografía endovenosa. Sin embargo, en ninguno de ellos fue posible medit las concentraciones plasmáticas de hormona patatiroidea $y$ 1-25 dihidroxivitamina D (dihidrocolecalciferol).

Todos los pacientes fueron sometidos a una dieta restringida en calcio (250 ing d día) y sodio ( $2 \mathrm{~g} \cdot \mathrm{di} a)$ por una semana, a partir de una pauta supervisada por la unidad de nutrición del hospital, para medir al octavo día, después de 12 h de régimen hídrico, el índice calciuria/cteatinina. Posteriomente, se indicó un régimen normal por 48 h y se admúnistró a las 8 A.M. del tercer día un desayuno con carga de calcio de $1 \mathrm{~g} \cdot 1,73 \mathrm{~m}^{2}$, en base a gluconato de calcio $10 \%$ diluido en $200 \mathrm{ml}$ de leche. Se recolectó la orina entre 8 y 12 h, para repetir la medición inicial de calciof creatinina.

La hipercalciuria absortiva se definió como un indice calcio/creatinina en ayunas menor de 0,21 , con ascenso después de la sobrecarga a más de 0.28 . La hipercalcturia renal se definió como un índice en ayunas de más de 0,21 , con ascenso postgluconato de calcio a más de 0,28 .

Las resultados se expresan como promedio (x) y desviación estándar; la evaluación estadística fue realizada mediante ensayo $t$ de Student para evaluación comparativa de grupos aleatorios y análisis de observaciones pereadas para lo comparación dentro de los grupos.

\section{RESULTADOS}

De acuerdo a la respuesta, 21 pacientes se clasificaron en el subtipo absortivo, 16 en el subtipo renal. En 13 pacientes la prueba no fue concluyente.

Los subgrupos hipercalciúricos estudiados no presentaron diferencias significativas respecto a edad y sexo (tabla 1). Los resultados de la prueba de restricción y sobrecarga se detallan en la tabla 2, donde se puede observar que el índice calcio/creatinina medido después de 7 días de restricción de calcio $y$ sodio es significativamente distinto entre los grupos absortivo y renal $(p<0,0005)$.

Después de la sobrecarga con gluconato de calcio se registra un significativo aumento del índice en el grupo absortivo ( $\mathrm{p}<0,0005$ respecto al valor previo); en el grupo renal el aumento en la excreción urinaria de calcio no es significativo respecto al valor en ayunas, marcando asi una diferencia radical respecto al subtipo absortivo. Ambos subtipos difieren del grupo control al administrar el calcio, ya que este último no registra aumento del indice cal-

Tabla 1

Caracteristicas clínicas de la población sometida a la prueba de restricción y sobrecarga de calcio

\begin{tabular}{lrrrrr}
\hline $\begin{array}{l}\text { Tipo de } \\
\text { hipercalciuria }\end{array}$ & $\mathbf{n}$ & $(\%)$ & \multicolumn{2}{c}{$\begin{array}{c}\text { Edad } \\
\text { (años) }\end{array}$} & \multicolumn{2}{c}{$\begin{array}{c}\text { Sexo (masculino) } \\
(\%)\end{array}$} \\
\hline Absortiva & 21 & $(42)$ & 9,9 & 12 & $(63)$ \\
Renal & 16 & $(32)$ & 11,8 & 10 & $(66)$ \\
No concluyente & 13 & $(26)$ & 9,3 & 7 & $(70)$ \\
Control & 15 & $(100)$ & 10,6 & 11 & $(73)$ \\
\hline
\end{tabular}

Tabla 2

Resultados de la prueba de restricción y sobrecarga de calcio

Tipo de

hipercalciuria

Absortiva

Renal

No concluyente

Control
Indice calciuria/creatininuria

postrestricción

postsobrecarga

p

$\begin{array}{ccc}0,44 \pm 0,09 & <0,0005 \\ 0,43 \pm 0,19 & \text { NS } \\ 0,16 \pm 0,04 & - \\ 0,20 \pm 0,07 & <0,025\end{array}$

$\overrightarrow{\mathrm{N} S}=$ No significativo. 
cio/creatinina por sobre el valor limite normal fijado. El ascenso entre los 2 indices medidos para el grupo control (pre y postcarga) es mayor que otros valores encontrados en la literatura $(p<0,025)$, lo que puede reflejar el pequeño número de pacientes evaluados y la presencia, entre ellos, de seis pacientes con índice postsobrecarga mayor a 0,21 . No es posible establecer una adecuada clasificación para 13 pacientes debido a su errático comportamiento frente a la prueba. En dos de ellos el indice es mayor que 0,21 después de la restricción y menor que 0,21 después de la sobrecarga. Los otros 11 presentan un patrón normal para ambos valores de calciof creatinina.

\section{COMENTARIO}

El pediatra se ve enfrentado con cierta frecuencia al intrincado diagnóstico etiológico de la urolitiasis y la hematuria, enfermedades en las cuales se ha ido asignando un rol de creciente importancia a la hipercalciuria idiopática ${ }^{1-6}$.

Los limites normales propuestos originalmente para la prueba aquí mencionada ${ }^{7}$ han sufrido modificaciones al ser evaluados en niños, con criterios más exigentes que fijan el límite normal en ayunas del índice calcio/creatinina en $0,21 \mathrm{y}$ postsobrecarga en $0,28^{5,16,18-20}$.

Sin embargo, el clásico y tranquilizador modelo fisiopatologico estudiado por Pak no ha sido corroborado por otros autores, que han objetado la utilidad de la prueba y encontrado diferencias con la secuencia fisiopatológica original, postu. lando la existencia de una gama en la cual las formas absortivas y renales puras son extremos de un trastorno renal primario, probablemente en el metabolismo de la vitamina D, en la reab. sorción tubular de fósforo o ambos ${ }^{6,12-15}$. Así, se ha confirmado la existencia de hipercalciuria en ayunas en pacientes portadores de formas absortivas a partir de la simulación de excesos de vitamina $D^{15}$, como también se han evidenciado hipercalciurias idiopáticas rena. les con niveles normales de hormona paratiroidea y aumento de las concentraciones plasmáticas de vitamina $D^{14}$. En otros estudios no se encontraron diferencias en los niveles plasmáticos de $\mathrm{PTH}$, vitamina $D$ y adenosina monofosfato cíclico urinario al estudiar en grupo de pacientes con hipercalciuria idjopática separados en subtipos absortivo y renal mediante restricción de cal- cio $^{19}$. Todos estos elementos de apoyo y duda han sido previamente delineados por Pak en su revisión del tema ${ }^{6}$. Nuestro estudio confirma la existencia de dos grupos distintos de pacientes hipercalciúricos al enfrentarlos a una restricción y sobrecarga de calcio, hecho de evidente interés terapéutico; sin embargo, la presencia de ciertos pacientes en los que la prueba no es concluyente indica que la separación propuesta no es absolu. ta. Además, el subgrupo de tipo renal parece dividido en dos poblaciones que difjeren en su respuest a a la prueba aplicada; la primera, presen. ta aumento de la excreción urinaria de calcio después de la sobrecarga, en tanto que en los demás la hipercalciuria disminuye frente a la administración de calcio oral, lo que permite suponer que correspondan a una forma primariamente perdedora de fósforo a nivel renal, cuya caracterización definitjva, igual que para el resto de los pacientes, requiere de un estudio más completo que incluye PTH y concentración de vitamina $D$ en el plasma.

La influencia de la dieta en la excreción urinaria de calcio ha sido recientemente revisada en forma exhaustiva, concluyéndose que existen condiciones que generan hipercalciuria, tales como las dietas con alto contenido de sodio, de calcio y de proteínas $\mathrm{s}^{8,19,21,22}$.

El manejo del paciente portador de hipercalcjuria idiopática debería basarse, en los casos en que sea posible su caracterización, en restriccion dietética de calcio y sodio para el subtipo absor. tivo o en administración de medicamentos que disminuyan la calciuria en los casos de origen renal, lo que se consigue eficientemente con hidroclorotiazida. La mala jndicación terapéutica de cualquiera de estas dos medidas puede producir desbalance de calcio en el organismo, lo que es un serio trastomo para un paciente en crecimiento ${ }^{5,23-25}$,

Falta mucho para afirmar que este tema ha sido aclarado. El seguimiento periódico y el estudio sistemático de los pacientes, con mejores recursos de laboratorio, hará seguramente posible responder algunas de las cuestiones pendientes para manejar correctamente al paciente pediátrico portador de este trastorno.

\section{RESUMEN}

Se evaluaron 50 pacientes portadores de hipercalciutia idiopática pesquisados a través 
de hematuria o litiasis y a 15 niños sanos controles. Todos ellos fueron sometidos a una prueba de restricción y sobrecarga de calcio, observándose tres distintos tipos de respuesta. El primer grupo ( $\mathrm{n}=21 ; 42 \%)$ presentó índices de calciuria/creatininuria postrestricción de cal. cio inferiores a 0,21 y mayores de 0,28 después de la sobrecarga, lo que permitió catalogarlos de hipercalciuria absortiva. El segundo grupo $(n=16 ; 32 \%)$ present 6 ambos índices sobre 0,21 , por lo que se catalogaron como subtipo renal, $y$ en el tercero $(n=16 ; 26 \%)$ las cifras obtenidas no permitieron su inclusión dentro de alguno de los dos subtipos precedentes. Nuestros resultados permiten concluir que mediante la aplicación ambulatoria de una prueba de restricción y sobrecarga de calcio es posible separar a los pacientes portadores de hipercalciuria idiopática en los subgrupos absortivo y renal, lo que resulta una valiosa ayuda desde el punto de vista terapéutico. Es necesario realizar estudios más completos para explicar la existencia de un subgrupo de errático comportamiento frente al ensayo, que probablemente representa formas intermedias entre los subtipos descritos.

(Palabras clave; hematuria, litiasis urinaria, cálculos urinarios, hipercalciuria idiopática, indice urinario de calcio/creatinina).

\section{REFERENCIAS}

1. Stapleton F.B., Roy III Sh., Noe H.N, Jerkins J.: Hypercalciuria in children with Hematuria. N Engl J Med 1984; 310: 1345-1348.

2. Kalis A., Travis L.B., Broukard B.H.: The association of idiopathic hypercalciurja and asyntomatic gross hematuria in children. J Pediatr 1981; 99: 716-719.

3. Roy III Sh., Stapleton F.B., Noe H.N., Jerkins J.: Hematuria preceding renal calculus formation in children with hypercalciuria. J Pediatr 1981; 99: $712-715$.

4. Langman C.B., Moore E.S. Hypercaleiuria in clinical pediatric. Clin Pediatr 1984; 23: 135-137.

S. Stapleton F.B., Mc Kay Ch. P., Noe H.N.: Utoli thiasis in children: The role of hypercalciuria. Pediatr Ann, 1987; 16: 980-982.

6. Pak Ch. Y. $C_{n}$. Kidney Stones. In: Williams Textbook of Endoctinology. Philadelphia. W. B. Saunders Co., 1985; 1256-1273.
7. Pak Ch. Y. C., Kaplan R., Bone H. et al.: A simple test for the diagnosis of absortive, resortive and renal hypercalciurias. N Engl J Med 1975; 292: 497.500 .

8. Bordier Ph., Ryckewart A., Gueris J., Rassmusen H.: On the pathogenesis of so called Idiopathic Hypercalciuria. Am J Med 1977; 63: 398-408.

9. Coe F.L., Breshinsky D.A.: Pathophysiology of Hypercalciuria. Am J Physiol 1984; 247: fi-f13.

10. Pak Ch. Y.C., Okata M., Lawrence E.C. Snyder $W .:$ The hypercalciurias: causes, parathyroid function and diagnosis criteria. J Clin Invest $1974 ; 54: 387.400$.

11. Santos F.. Suárez D., Málaga S., Crespo M.: Idiopathic hypercalciuria in children pathophysiologic considerations of renal and absortive subtypes. J Pediatr 1987;110: 238-243.

12. Lein J.W., Keane P.M.: Limitation of the oral calcium loading test in the management of the recurrent calcarcous renal stone former. Aim J Kidney Dis 1983; 3: 76-78.

13. Hellen J.E., Konrak J.E., Lau Y.: Potential pitfalls of the calciom creatinine ratio and urinary cyclic adenosine monophosphate excretion in the differential điagnosis of idiopathic hypercalciuria. J Urol 1984;131: 911.913.

14. Stapleton F.B., Langman C.B., Bittle J., Miller $L . A$.: Increased serum concentrations of $1,25(\mathrm{OH})_{2}$ vitamin D in ehildren with fasting hypercalciuria. J Pediatr 1987; 110: 234-237.

15. Broadus A.E., Erickson S.B., Germer J.M. et al. An experimental human model of 1-25 dihydroxyvitamin D mediated hypercalciuria. J Clin Endocrinol Metab 1984: 59: 202-206.

16. Stopleton F.B. Noe H.N., Jerkins J., Roy III Sh. Uninary excretion of calcium following an oral calcium loading test in healthy children. Pediatrics 1982;69: 594-597.

17. Lein J., Keqne P.M.: Urinary cAMP and calcium excretion in the fasting state and the response to oral calcium loading in patients with calcium nephrolithiasis. J Urol 1983; 129: 401-404.

18. Krusse K., Kracht $U_{\text {., Knisse V.: Reference values }}$ for urinasy calcium excretion and screening for hypercalciuria in children and adolescents. Eur 3 Pediatr 1984: 143: 25-31.

19. Coe F.L., Fauns L.J., Crochett T. et al.: Effects of low calcium diet on calciun excretion, parathyroid function and serum 1,25 Vit D levels in nine patients with idiopathic hypercalciuria and five nomal subjets. Am J Med 1982; 72: 25-32.

20. Sutton $\boldsymbol{R}$.A.: Disorders of renal calcium excretion. Kidney Int 1983; 23: 665-673.

21. Breslaw N.A., Mc Guire J.L., Zcewekh J.E., Pak Ch. Y.C.: The role of dietary sodium on renal excretion and intestinal absortion on calcium and Vitamin D metabolism. J Clin Endocrinol Metab 1982; 55: 369-373.

22. Goldfard S.: Dietary factors in the pathogenesis and prophylaxis of calcium nephrolithiasis. Kidney Int 1988; 34: 544-555. 